\title{
A Prospective Study on Lactation Status and Breastfeeding Challenges of Mothers Giving Birth to Preterm Infants
}

\section{Dingding Dong}

Peking University First Hospital https://orcid.org/0000-0003-1306-6589

\section{Xifang Ru}

Peking University First Hospital

\section{Xiaofang Huang}

Peking University First Hospital

\section{Tian Sang}

Peking University First Hospital

\section{Shan Li}

Peking University First Hospital

\section{Ying Wang}

Peking University First Hospital

Qi Feng ( $\square$ fengqizf@126.com)

Peking University Hospital https://orcid.org/0000-0001-7321-6134

\section{Research}

Keywords: Infant, premature, breastfeeding, Lactogenesis II

Posted Date: October 19th, 2020

DOl: https://doi.org/10.21203/rs.3.rs-92675/v1

License: (1) This work is licensed under a Creative Commons Attribution 4.0 International License. Read Full License 


\section{Abstract}

Background

Mothers of preterm infants face challenges in breastfeeding. The aim of the study was to investigate lactation status and challenges in breastfeeding faced by mothers of preterm infants.

Methods

Mothers giving birth to preterm infants between May $26^{\text {th }} 2018$ and October $31^{\text {st }} 2018$ were enrolled. Lactation status and challenges in breastfeeding on day 7 postpartum, at discharge of infants, 2 weeks post-discharge, and 3 months of corrected age (CA $3 \mathrm{~m})$ were collected by questionnaires.

Results

Seventy mothers were enrolled, and $51.4 \%$ had delayed lactogenesis II. Multivariate logistic regression analysis revealed that cesarean section ( $\mathrm{OR}=3.368, p=0.032)$, older maternal age $(\mathrm{OR}=1.189, p=0.008)$, and primiparity $(\mathrm{OR}=5.091, p=0.012)$ were significant independent predictors of delayed lactogenesis II. Mothers with delayed lactogenesis II had significantly lower milk output (day 7 postpartum: $160.0 \mathrm{~mL}$ vs. $300.0 \mathrm{~mL}$, $p=0.001$; at discharge: $425.0 \mathrm{~mL}$ vs. $612.5 \mathrm{~mL}, p=0.005$ ) and lower proportion of exclusive breastfeeding (at discharge: $38.9 \%$ vs. $70.6 \%, p=0.008$; CA $3 \mathrm{~m}: 12.5 \%$ vs. $33.3 \%, p=0.050$ ). The ROC showed that milk output $>190 \mathrm{~mL} / \mathrm{d}$ and $>245 \mathrm{~mL} / \mathrm{d}$ on day 7 postpartum significantly predict milk output $\geq 300 \mathrm{~mL} / \mathrm{d}$ at discharge and exclusive breastfeeding at post-discharge two weeks, respectively. Insufficient human milk was the top reason for breastfeeding discontinuation at CA $3 \mathrm{~m}$, and mother's own milk $\geq 50 \%$ of total milk uptake at postpartum two weeks $(\mathrm{OR}=5.303, p=0.007)$ was an independent predictor of continuous breastfeeding at CA $3 \mathrm{~m}$. Feeding complications in infants, poor breastfeeding techniques, and low milk output were the top challenges in breastfeeding.

Conclusion

Interventions to improve early postpartum lactation and breastfeeding techniques may increase breastfeeding adoption in mothers of preterm infants.

\section{Background}

Preterm infants benefit from breastfeeding in many ways, including shorter hospital stay ${ }^{[1-3]}$, lower incidences of severe complications including necrotizing enterocolitis ${ }^{[3-5]}$ and bronchopulmonary dysplasia ${ }^{[2]}$, better developmental outcomes, and lower incidence of rehospitalization ${ }^{[6]}$. Therefore, human milk has been recognized as the ideal food for preterm infants in neonatal intensive care units (NICUs). However, the prevalence of breastfeeding in preterm infants varies greatly worldwide.

In the United States, $75 \%$ of NICU preterm infants were breastfed in $2015^{[7]}$. In Europe, $58.5 \%$ of preterm infants (gestational age, GA $<32$ weeks) received human milk at discharge, and $42.6 \%$ of the infants in the study received donor milk ${ }^{[8]}$. In China, the prevalence of breastfeeding varies widely in different hospitals ${ }^{[9]}$. 
Hei et al investigated the preterm infants (GA 28-35 weeks) of 9 NICUs in China and found a breastfeeding prevalence of only $34.9 \%$ in $2017^{[10]}$. In the NICU of the Peking Union Medical College Hospital, $65 \%$ of preterm infants (GA<32 weeks) were breastfed in $2011^{[11]}$. In Nanjing Maternity Hospital Affiliated to Nanjing Medical University, human milk from a donor milk bank was available for all of the infants with very low or extremely low birth weight ${ }^{[12]}$.

Parents usually participate in breastfeeding education and training during pregnancy, however, the stress associated with preterm delivery often disrupts their original breastfeeding plan. Although the breastfeeding of preterm infants improved a lot in recent years, it remains to be a big challenge for mothers who give birth to sick infants and thus have to be separated from their infants in NICU. In addition, to reduce the risk of infection for preterm infants, healthcare professionals have to limit the time and frequency of the mothers' visits to their infants in the NICU. Preterm infants admitted to NICU often have critical conditions and low birth weight, and their mothers' milk is definitively the best food for them. Thus, to support breastfeeding, hospitals encourage mothers to feed their preterm infants with their own expressed milk. Expressed milk can be sent to NICU frequently. Currently, human milk bank has not been commonly practiced in China ${ }^{[13]}$. Thus, preterm infants in China mostly rely on their mother's own milk (MOM).

In order to understand the challenges in breastfeeding faced by mothers giving birth to preterm infants and develop effective interventions to improve breastfeeding in preterm infants in NICU, we aimed to investigate the lactation status of mothers of preterm infants.

\section{Methods}

\section{Study Design and Setting}

This was a prospective cohort study. The study protocol was approved by the Institutional Review Board of the hospital (project identification code: 2018-88; date of approval: May $23^{\text {rd }}, 2018$ ). Written informed consent was obtained from all participating mothers.

\section{Study Participant Inclusion and Exclusion Criteria}

Preterm infants' mothers were recruited between May $26^{\text {th }}$ and October $31^{\text {st }}, 2018$ on day 1 to day 4 of infants' NICU entry, and they were followed up till 3 months of corrected age (CA). The inclusion criteria were: (i) mothers giving birth to infants before 37 weeks of GA and their infants were admitted to the NICU; (ii) mothers intended to breastfeed their infants. The exclusion criteria were: (i) mothers did not sign the informed consent form; (ii) infants died; infants were transfer to other departments; or mothers took their infants home against medical advice

\section{Infant Feeding Procedure}

Enteral feeding was started as soon as the infant's condition getting stable and no contraindications. The expressed mother's own milk was prioritized. The mothers were encouraged to express milk postpartum as soon as their conditions became stable, and their expressed milk was sent to the NICU directly when the 
mothers stayed in the obstetric ward. After the mothers were discharged from the hospital, their expressed milk was frozen at home and then transported to the NICU by their family members. The infants were fed with MOM by either gastric tube or bottle during their hospital stay by healthcare staff. Human milk fortifier (HMF) was added if the total volume of consumed human milk reached $50 \mathrm{~mL} / \mathrm{kg} / \mathrm{d}$ in infants with a $\mathrm{GA}<32$ weeks or birth weight $<2000 \mathrm{~g}$. All the feeding information was documented daily in medical records, including feeding volume, human milk volume, and tolerance. Direct-breastfeeding was recommended after the infant no longer need full strength HMF after discharge. If MOM was insufficient, infants were fed with additional preterm formula or post-discharge formula. The criteria to discharge the infants were that infants matured, reached corrected GA $\geq 34$ weeks, had a bodyweight $>2000 \mathrm{~g}$, had a daily milk intake volume > $150 \mathrm{~mL} / \mathrm{kg} / \mathrm{d}$, were able to suck and drink all milk orally, and had stable vital signs. The mothers and their infants were required to come back for regular post-discharge follow-up examination until CA (corrected age) of 2 years. Infant's information, including perinatal data, feeding, and medical condition in hospital and follow-up clinics, were collected from the hospital electric medical record systems.

\section{Questionnaire Survey for Participating Mothers}

Participating mothers were required to complete questionnaires on lactation and breastfeeding practice on day 7 postpartum, at discharge day of infants, 2 weeks after the discharge of infants, and 3 months of CA of infants either by filling the questionnaires by themselves or by telephone conversation.

In general, the questionnaires collected mothers' input on expressing milk practice, milk output volume, and challenges in breastfeeding. In particular, on day 7 postpartum, the questionnaire collected the information regarding milk output volume in the past 24 hours, the type of pump that was used, milk expression frequency, and the day of lactogenesis II onset. At the discharge of infant, the questionnaire, which was completed in 48 hours before the discharge, collected data of expressed milk volume in the past 24 hours, milk expression frequency, and the challenges in breastfeeding during the infant hospital stay. The questionnaires that were conducted 2 weeks after infant discharge and CA of 3 months collected infants' feeding method, mothers' perception of milk volume changes, reasons to discontinue breastfeeding, and challenges in breastfeeding at home.

\section{Definitions}

Breastfeeding was defined as feeding infants with either partial or exclusive human milk in 24 hours of each time point. Human milk volume was defined as the volume of human milk in 24 hours. Human milk proportion was the proportion of human milk in the total milk consumed by infants in 24 hours. Expressed milk volume was the total expressed human milk volume in 24 hours. Lactogenesis II onset was defined as the day when mothers were able to express $20 \mathrm{~mL}$ milk (the total amount from both sides) for consecutive three times. Delayed lactogenesis II onset was defined as the failure of lactogenesis II onset within postpartum 4 days. Feeding complications includes abdominal distension, vomiting, diarrhea, hematochezia, and poor weight gain.

\section{Statistical Analysis}


Continuous variables with non-normal distribution are presented as median ( $1^{\text {st }}$ quartile, $3^{\text {rd }}$ quartile). Categorical variables are presented as the number of cases and percentages. Chi-square and the MannWhitney $U$ test were performed to compare variables of different groups and analyze independent predictors. The receiver operating characteristic (ROC) curve was prepared to use milk output volume on day 7 postpartum to predict later breastfeeding status. Logistic regression analyses were performed to identify factors associated with delayed lactogenesis II onset and with continuation of breastfeeding at CA of 3 months. The statistical significance level was set at $p<0.05$ for all analyses. IBM SPSS Statistics Version 22.0 (SPSS, Chicago, IL, USA) was used for all the statistical analyses.

\section{Results}

\section{Participant Flow Chart and Clinical Characteristics}

The participant flow chart is presented in Figure 1. A total of 157 preterm infants with a GA $<37$ weeks from 124 mothers were admitted to the NICU between May $26^{\text {th }}$ and October $31^{\text {st }}, 2018$. Of the 124 mothers, 30 were excluded for the following reasons: did not intend to breastfeed $(n=12)$, did not sign the informed consent $(n=1)$, neonatal death $(n=2)$, infants being transfer to other departments $(n=3)$, or neonatal nonmedical discharge $(n=12)$. A total of 94 eligible mothers (Full analysis set) participated in the study. During the study, 24 mothers failed to complete the questionnaire during the infant hospital stay. Data from 70 mothers (per protocol set) were analyzed.

Table 1 displays the clinical characteristics of mothers The median age of the 70 mothers was 34.0 (31.0, 36.0 ) years. Of the 70 mothers, $64.3 \%$ (45/70) were primiparous; $62.9 \%$ (44/70) had pregnant complications, including hypertensive disorders of pregnancy, gestational diabetes mellitus, thyroid disease, intrahepatic cholestasis, appendicitis, and glomerulonephritis; 70.0\% (49/70) gave birth by cesarean section. The clinical characteristics were similar between the full analysis set and per protocol set (Table 1). The subsequent analyses were performed on the per protocol set. Of the 94 infants, 52.1\% (49/94) were twins; the median GA and birth weight were $32.9(31.2,34.0)$ weeks and $1770(1477.5,2042.5) \mathrm{g}$, respectively; the median of NICU stay was $25.0(16.0,35.5)$ days. At 3 months of CA of infants, 62 mothers with their 81 preterm infants came back to the follow-up examination.

Table 1. Clinical Characteristics of Mothers 


\begin{tabular}{|llll|}
\hline & \begin{tabular}{llll|}
\hline \\
\end{tabular} & $n=94$ & $p^{*}$ \\
\hline Age (year) & $34.0(31.0,36.0)$ & $34.0(31.0,36.0)$ & 0.740 \\
\hline Complications during pregnancy, $\mathrm{n}(\%)$ & $57(60.6)$ & $44(62.9)$ & 0.773 \\
\hline Cesarean section, $\mathrm{n}(\%)$ & $67(71.3)$ & $49(70.0)$ & 0.859 \\
\hline Gestational age (week) & $32.6(31.0,34.1)$ & $32.8(30.9,34.0)$ & 0.991 \\
\hline Primiparous, $\mathrm{n}(\%)$ & $61(64.9)$ & $45(64.3)$ & 0.936 \\
\hline Twin, $\mathrm{n}(\%)$ & $30(31.9)$ & $26(37.1)$ & 0.485 \\
\hline
\end{tabular}

Note. Data are represented as number (\%) or median (interquartile); * $p$-value is based on the results of a Mann-Whitney $\mathrm{U}$ test for continuous variables and a chi-square test for categorical one.

\section{Lactation Status}

Survey of the types of breast pump used by mothers showed that $65.7 \%(46 / 70), 32.9 \%(23 / 70)$, and $1.4 \%$ $(1 / 70)$ of the mothers used unilateral electric, bilateral electric, and manual breast pumps, respectively. The median of expressing frequency per 24 hours was $7.5(5.5,9.5)$ times and $6.0(5.0,8.0)$ times on day 7 postpartum and at infant discharge, respectively (Figure 2A).

The median daily milk output was $220.0(110.0,400.0) \mathrm{mL}$ on day 7 postpartum and then increased to $525.0(277.5,762.5) \mathrm{mL}$ at discharge $(p<0.001)$. On day 7 postpartum, $65.7 \%$ of the mothers expressed more milk than their infants' uptake, and this proportion raised to $68.6 \%$ at the discharge of infants. The proportions of the mothers showing increased milk output at discharge, post-discharge 2 weeks, and CA of 3 months compared with the previous time point were $81.4 \%, 50.0 \%$, and $33.9 \%$, respectively (Figure 2B), indicating that fewer mothers had increased milk output and an increasing number of mothers produced less milk as time went by. At CA of 3 months, $53.2 \%$ of the mothers produced less milk than at postdischarge 2 weeks.

The ROC analysis found that milk output volume on day 7 postpartum reaching $190 \mathrm{~mL} / \mathrm{d}$ was an optimal threshold for predicting milk output volume $>300 \mathrm{~mL} / \mathrm{d}$ at discharge (sensitivity $72.5 \%$, specificity $73.7 \%$, area under the curve [AUC] 0.772; $p=0.001$, Figure 2C). The milk output volume of $245 \mathrm{~mL} / \mathrm{d}$ on day 7 postpartum predicted the possibility of exclusive breastfeeding at post-discharge two weeks (sensitivity 72.7 $\%$, specificity $65.9 \%$, AUC $0.725 ; p<0.001$ ) (Figure 2 C).

Of the 70 mothers, $51.4 \%(36 / 70)$ and $20.0 \%$ (14/70) failed to achieve lactogenesis II within postpartum 4 and 7 days, respectively. Compared with the mother with normal lactogenesis II onset, the mothers with delayed lactogenesis II onset showed a significantly higher proportion of cesarean section ( $80.6 \%$ vs. $58.8 \%$, $p=0.047)$ and a higher percentage of the primiparous mothers $(75.0 \%$ vs. $52.9 \%, p=0.054)$. Maternal age, complications during pregnancy, singleton, infant gestational age, and infant Apgar score and mechanical ventilation duration were similar between the normal and delayed groups (Table 2). Multivariate binary 
logistic regression analysis adjusted by twin, pregnant complications, GA, mechanical ventilation $>3$ days, and Apgar score at postpartum 5 minutes revealed that cesarean section (OR=3.368; 95\%Cl: 1.043-10.869, $p=0.032)$, older maternal age ( $\mathrm{OR}=1.189 ; 95 \% \mathrm{Cl}: 1.015-1.393, p=0.008)$, and primiparous status $(\mathrm{OR}=5.091 ; 95 \% \mathrm{Cl} 1.525-16.994, p=0.012)$ were significant independent predictors of delayed lactogenesis II onset.

The mothers with delayed lactogenesis II onset had significantly lower daily mild output on day 7 postpartum $[160.0(88.1,287.5) \mathrm{mL}$ vs. $300.0(187.5,550.0) \mathrm{mL}, p=0.001]$ and discharge day [425.0 (200.0, $600.0)$ vs. $612.5(465.0,850.0), p=0.005$ ] than the mother with normal onset (Table 2$)$. In addition, the exclusive breastfeeding rate at discharge ( $38.9 \%$ vs $70.6 \%, p=0.008)$ and CA of 3 months $(12.5 \%$ vs $33.3 \%$, $p=0.050$ ) were also significantly lower in the delayed group (Table 2).

Table 2. Comparison of the Characteristics and Breastfeeding Status of Delayed versus Normal Lactogenesis II Onset Groups

\begin{tabular}{|c|c|c|c|}
\hline & Delayed group & Normal group & $p^{*}$ \\
\hline Mothers' characteristic & $n=36$ & $n=34$ & \\
\hline Age years, Median (min, max) & $34.0(31.0,36.0)$ & $34.0(32.0,36.0)$ & 0.415 \\
\hline Complications during pregnancy n (\%) & $26(72.2)$ & $18(52.9)$ & 0.095 \\
\hline Gestational age (week), Median (min, max) & $32.4(30.3,33.7)$ & $33.1(31.2,34.3)$ & 0.055 \\
\hline Cesarean section, n (\%) & $29(80.6)$ & $20(58.8)$ & 0.047 \\
\hline Primiparous, n (\%) & $27(75.0)$ & $18(52.9)$ & 0.054 \\
\hline Twin, n (\%) & $16(44.4)$ & $10(29.4)$ & 0.193 \\
\hline \multicolumn{4}{|l|}{ Infants characteristic ${ }^{1}$} \\
\hline Apgar score at $5^{\prime} \otimes 7, \mathrm{n}(\%)$ & $1(2.8)$ & 0 & $1.000^{2}$ \\
\hline Mechanical ventilation $\geq 3$ days, $n(\%)$ & $3(8.3)$ & $4(11.8)$ & 0.632 \\
\hline \multicolumn{4}{|l|}{ Daily milk output (mL) } \\
\hline On postpartum day 7 , median (interquartile) & $160.0(88.1,287.5)$ & $300.0(187.5,550.0)$ & 0.001 \\
\hline At discharge, median (interquartile) & $425.0(200.0 \otimes 600.0)$ & $612.5(465.0,850.0)$ & 0.005 \\
\hline \multicolumn{4}{|l|}{ Breastfeeding status } \\
\hline Exclusive breastfeeding at discharge, $\mathrm{n}(\%)$ & $14(38.9)$ & $24(70.6)$ & 0.008 \\
\hline Breastfeeding at CA 3 months, $n(\%)^{3}$ & $12(37.5)$ & $14(46.7)$ & 0.465 \\
\hline Exclusive breastfeeding at CA 3 months, $n(\%)^{3}$ & $4(12.5)$ & $10(33.3)$ & 0.050 \\
\hline
\end{tabular}


Note. Data are represented as number (\%) or median (interquartile); * $p$-value is based on the results of a Mann-Whitney $U$ test for continuous variables and a chi-square test for categorical one.

${ }^{1}$ Mothers with twin preterm infants were counted if either of the twins had the following characteristics.

${ }^{2}$ Fisher's test was used.

${ }^{3}$ At CA of 3 months, 62 mothers participated in the follow-up; of them, 32 had delayed lactogenesis II onset and 30 had normal onset.

\section{Breastfeeding Status at Discharge and Post-discharge}

The proportions of infants receiving breastfeeding (70.4\%) and exclusive breastfeeding (33.3\%) at CA of 3 months reduced compared with those at discharge (breastfeeding: $78.7 \%$, exclusive breastfeeding $48.9 \%$ ) and post-discharge two weeks (breastfeeding: $83.0 \%$, exclusive breastfeeding $48.9 \%$, Table 3 ), although the differences were not statistically significant. Of the 94 infants, 21.3\% (20/94) were fed with formula at discharge, and the top reason for discontinuing breastfeeding was infant clinical condition (14/20, Table 3), including one infant having cytomegalovirus (CMV) infection and 13 having gastrointestinal (GI) problems, including hematochezia, abdominal distension, diarrhea, and necrotizing enterocolitis (6 of the 13 infants resumed breastfeeding two weeks after discharge). At post-discharge two weeks, $17.0 \%$ (16/70) infants discontinued breastfeeding, and the top reason was also infant clinical condition (11/16, 10 cases of GI problems and 1 case of CMV infection). At CA of 3 months, 29.6\% (24/62) infants discontinued breastfeeding, and the most common reason was insufficient human milk (14/24, Table 3). Compared with the proportion of infant discontinuing breastfeeding because of insufficient human milk at discharge (3/94, $3.2 \%)$ and post-discharge two weeks (5/94, 5.3\%), a significantly higher proportion (14/81, $17.3 \%)$ of the infant at CA of 3 months discontinued breastfeeding for the same reason $(p<0.0167$, Table 3$)$.

Table 3. Breastfeeding status at discharge, post-discharge two weeks, and CA of 3 months 


\begin{tabular}{|c|c|c|c|c|}
\hline & $\begin{array}{l}\text { Discharge, } \mathrm{n} \\
(\%)\end{array}$ & $\begin{array}{l}\text { Two weeks post-discharge, } \\
\mathrm{n}(\%)\end{array}$ & $\begin{array}{l}\text { CA } 3 \text { months, } n \\
(\%)\end{array}$ & $p^{*}$ \\
\hline Mothers, $n$ & 70 & 70 & 62 & - \\
\hline Infants, $n$ & 94 & 94 & 81 & - \\
\hline Breastfeeding, n (\%) & $74(78.7)$ & $78(83.0)$ & $57(70.4)$ & 0.130 \\
\hline $\begin{array}{l}\text { Exclusive breastfeeding, } \\
\mathrm{n}(\%)\end{array}$ & $46(48.9)$ & $46(48.9)$ & $27(33.3)$ & 0.061 \\
\hline Formula feeding, n (\%) & $20(21.3)^{a}$ & $16(17.0)$ & $24(29.6)$ & 0.130 \\
\hline \multicolumn{5}{|c|}{ Reasons for discontinuing breastfeeding } \\
\hline Infants' factors & $\begin{array}{l}14(14 / 20 \\
70.0)\end{array}$ & $11(11 / 16,68.8)$ & $\begin{array}{l}10(20 / 24 \\
41.7)\end{array}$ & 0.814 \\
\hline Insufficient human milk & $\begin{array}{l}3(3 / 20 \\
15.0)\end{array}$ & $5(5 / 16,31.3)$ & $\begin{array}{l}14(14 / 24 \\
58.3)^{\mathrm{cd}}\end{array}$ & 0.001 \\
\hline Others & $\begin{array}{l}3(3 / 20 \\
15.0)^{b}\end{array}$ & 0 & 0 & - \\
\hline
\end{tabular}

Note. Data are represented as number (\%); * $p$-value is based on the results of chi-square test. Bonferroni adjustment was made at $\mathrm{p}=0.0167(0.05 / 3)$.

${ }^{a}$ Three infants were formula-fed during hospitalization because their mothers expressed very little milk and the families did not transport it to the hospital, and they started breastfeeding post-discharge.

b Three infants were breastfed during hospitalization, but their family did not transport milk to the hospital during the last three days of the infant hospital stay, but they all resumed breastfeeding post-discharge.

${ }^{\mathrm{c}}$ Insufficient human milk rate higher than discharge.

${ }^{d}$ Insufficient human milk rate higher than two weeks post-discharge.

We then investigated the factors that could be associated with continuous breastfeeding at CA of 3 months. Of the 62 mothers, $46(46 / 62,74.2 \%)$ practiced breastfeeding and $16(25.8 \%)$ did not at CA of 3 months (Table 4). Exclusive breastfeeding at discharge (63.0\% vs. $31.3 \%, p=0.028)$, infants receiving $M O M \geq 50 \%$ of total milk uptake at postpartum two weeks $(76.1 \%$ vs. $37.5 \%, p=0.005)$, and direct feeding at postpartum two weeks $(73.9 \%$ vs. $43.8 \%, p=0.028)$ were significantly associated with continuous breastfeeding at CA of 3 months (Table 4). Multivariable binary logistic regression analysis adjusted by twin, pregnant complications, cesarean section, primiparous status, breastfeeding at discharge, exclusive breastfeeding at discharge, $\mathrm{MOM} \geq 50 \%$ at postpartum two weeks, and direct feeding at postpartum two weeks, revealed that $\mathrm{MOM} \geq 50 \%$ at postpartum two weeks (OR=5.303; $95 \% \mathrm{Cl} 1.569-17.925, p=0.007)$ was an independent predictor of continuous breastfeeding at CA of 3 months. 
Table 4. Factors Associated with Continuous Breastfeeding at CA of 3 Months

\begin{tabular}{|llll|}
\hline & Breastfeeding & no breastfeeding & $p^{*}$ \\
\hline Twin, $\mathrm{n}(\%)$ & $n=46$ & $n=16$ & \\
\hline Breastfeeding at day 7, $\mathrm{n}(\%)$ & $14(30.4)$ & $8(50.0)$ & 0.159 \\
\hline Exclusive breastfeeding at day 7, $\mathrm{n}(\%)$ & $38(82.6)$ & $11(68.8)$ & 0.241 \\
\hline Breastfeeding at discharge, $\mathrm{n}(\%)$ & $16(34.8)$ & $3(18.8)$ & 0.231 \\
\hline Exclusive breastfeeding at discharge, $\mathrm{n}(\%)$ & $29(63.0)$ & $5(31.3)$ & $\mathbf{0 . 0 2 9}$ \\
\hline OMM $\mathbf{5 0 \%}$ at postpartum two weeks, $\mathrm{n}(\%)$ & $35(76.1)$ & $6(37.5)$ & $\mathbf{0 . 0 0 5}$ \\
\hline Direct feeding at two weeks postpartum, $\mathrm{n}(\%)$ & $34(73.9)$ & $7(43.8)$ & $\mathbf{0 . 0 2 8}$ \\
\hline
\end{tabular}

Note. Data are represented as number (\%); ${ }^{*} p$-value is based on the results of chi-square test. CA of 3 months: corrected age of three months; BPD: bronchopulmonary dysplasia; MOM: mother's own milk.

\section{Challenges in Continuing Breastfeeding}

Survey of the challenges in continuous breastfeeding faced by mothers found that difficulties in breastfeeding their infants and low milk volume were the predominant challenges during the infant hospital stay. At post-discharge 2 weeks, feeding complications in infants and poor breastfeeding techniques became the predominant challenges. At CA of 3 months, difficulties in breastfeeding their infants and low milk volume were the top two challenges (Table 5).

Table 5. Challenges in Breastfeeding during Hospitalization and after Post-discharge 


\begin{tabular}{|c|c|c|}
\hline & Challenges in breastfeeding & Response $\mathrm{n}(\%)$ \\
\hline \multicolumn{3}{|c|}{ During hospital stay $(n=70)$ * } \\
\hline \multirow[t]{3}{*}{ Associated with infants } & Difficulties in feeding & $22(31.4)$ \\
\hline & Feeding complications & $5(7.1)$ \\
\hline & Breastfeeding discontinuation for diseases & $3(4.2)$ \\
\hline \multirow[t]{4}{*}{ Associated with mothers } & Low human milk volume & $13(18.6)$ \\
\hline & Lack of breastfeeding knowledge & $9(12.9)$ \\
\hline & Difficulties in expressing milk & $3(4.2)$ \\
\hline & Difficulties in milk transportation & $2(2.9)$ \\
\hline \multicolumn{3}{|c|}{ Post-discharge 2 weeks $(n=70)$} \\
\hline \multirow[t]{2}{*}{ Associated with infants } & Feeding complications & $20(28.6)$ \\
\hline & Difficulties in feeding & $16(22.9)$ \\
\hline \multirow[t]{4}{*}{ Associated with mothers } & Poor breastfeeding techniques & $17(24.3)$ \\
\hline & Low human milk volume & $14(20.0)$ \\
\hline & Difficulties in expressing milk & $3(4.3)$ \\
\hline & Lack of breastfeeding knowledge & $4(5.8)$ \\
\hline \multicolumn{3}{|l|}{ CA of 3 months $(n=62)$} \\
\hline \multirow[t]{2}{*}{ Associated with infants } & Difficulties in feeding & $36(58.1)$ \\
\hline & Feeding complications & $26(41.9)$ \\
\hline \multirow[t]{5}{*}{ Associated with mothers } & Low human milk volume & $22(35.5)$ \\
\hline & Lack of breastfeeding knowledge & $14(24.7)$ \\
\hline & Difficulties in expressing milk & $6(9.7)$ \\
\hline & Lack of support from family & $3(4.8)$ \\
\hline & Lack of support from the workplace & $2(3.2)$ \\
\hline
\end{tabular}

Note. Data are represented as number (\%); CA of 3 months: corrected age of three months; *Infants were fed mainly by health care staff during hospitalization, and by parents after discharge.

\section{Discussion}

\section{Lactogenesis II Onset and Breastfeeding}


Previous studies have found that mothers giving birth to preterm infants experienced difficult lactation, including delayed lactation ${ }^{[14]}$, low milk output ${ }^{[15]}$, and premature discontinuation of lactation ${ }^{[16]}$. In early lactation, one of the important indicators is lactogenesis II onset, which marks the time when mothers begin to produce copious amounts of milk ${ }^{[17]}$.

Lactogenesis II usually occurs at postpartum 50-73 hours although the definition and measurement method of lactogenesis II onset may vary ${ }^{[14,17]}$. In our study, mothers were discharged from hospital earlier than their infants and they expressed milk at home during their infant hospital stay. Thus, we defined the day when mothers were able to express $20 \mathrm{~mL}$ milk (the total amount from both sides) for consecutive three times as the day of lactogenesis II onset ${ }^{[18]}$.

Delayed lactogenesis II is generally defined as a failure to achieve the initiation of lactogenesis II in postpartum 72 hours ${ }^{[19]}$. In this study, we found that $48.6 \%$ of the mothers of preterm infants achieved lactogenesis II within postpartum 4 days (72-96 hours) whereas the rest (51.4\%) failed. Some studies suggest that lactogenesis II onset occurred at postpartum 97.15 hours among preterm infants' mothers (infants birth weight $<1500 \mathrm{~g}$ ) ${ }^{[20]}$, and $36 \%$ of mothers of preterm infants (GA<37weeks) had delayed lactogenesis II onset (>postpartum 72 hours) ${ }^{[21]}$, which are similar to our results. Therefore, delayed lactogenesis II onset appears very common in mothers of preterm infants.

Delayed lactogenesis II onset can adversely affect breastfeeding in the later period. Previous studies found that mothers with delayed onset of lactogenesis II had lower milk output on day 7 postpartum than normal group [202.50 (164.75-280.00)vs $357.50(200.00-545.25)](p<0.001)^{[21]}$, and that mothers with delayed lactogenesis II onset were less likely to have exclusive breastfeeding postpartum 4 weeks (OR: 1.62; Cl $1.18-2.22)(p<0.01)^{[22]}$. We also found that the mothers with delayed lactogenesis II onset had significantly lower milk output and a lower proportion of exclusive breastfeeding. Delayed lactogenesis II

onset was also found to be associated with premature discontinuation of breastfeeding ${ }^{[23,24]}$. Although we observed a similar trend at infants' 3 months of corrected age in this study, the association was not statistically significant. ,

We found that cesarean section, older maternal age, and primiparous status were independent risk factors for delayed lactogenesis II onset, which are consistent with the results from previous studies [25][26][27][19, 28]. First birth and unscheduled cessation delivery might adversely affect lactogenesis and breastfeeding by increasing mothers' stress ${ }^{[28]}$.

\section{Predictive Values of Milk Output at Day 7 postpartum}

Our ROC analysis revealed that milk output $>190 \mathrm{~mL} / \mathrm{d}$ on day 7 postpartum predicted milk output $>300$ $\mathrm{mL} / \mathrm{d}$ at discharge and that output $>245 \mathrm{~mL} / \mathrm{d}$ on day 7 postpartum predicted exclusive breastfeeding at post-discharge two weeks. These results suggest that low milk output on day 7 postpartum might predict low milk output later and a low chance of later breastfeeding. Flacking et al ${ }^{[29]}$ found that insufficient human milk output by the end of the first week postpartum was associated with low breastfeeding rate in preterm infants in postpartum two months $(p=0.039)$ and four months $(p=0.001)$. Notably, Omarsdottir et al 
[30] used expressed milk output at postpartum two weeks to predict the breastfeeding status at postpartum 6 weeks and discharge. We used milk output at day 7 postpartum for the prediction. Our method could effectively identify mothers with lactation difficulties at earlier stage and thus guide healthcare professionals to intervene earlier to improve mothers' lactation and breastfeeding.

\section{Breastfeeding Status at CA of 3 Months}

In this study, at CA of 3 months, $70.4 \%$ of the infants were continuously breastfed, and only $33.3 \%$ were breastfed exclusively. Meio et al reported that breastfeeding and exclusive breastfeeding rate in preterm infants (GA<33 weeks) at CA of 3 months was $62.4 \%$ and $4.3 \%$, respectively ${ }^{[31]}$, which are lower than our results. The continuous patient education in our institution and higher GA of the preterm infants may contribute to the higher breastfeeding rate at CA of 3 months in this study.

We found at CA of 3 months, 53.2\% of the mothers reporting a decreased human milk output, a lower exclusive breastfeeding rate (33.3\%) in the infants, and a higher proportion of infants discontinuing breastfeeding because of insufficient human milk (58.3\%), which are similar to the results from previous studies ${ }^{[16,32]}$. Because long-lasting and a high proportion of human milk are beneficial to the nervous system development and the overall health of preterm infants ${ }^{[33]}$, we believe induction of early lactogenesis Il onset and continuous breastfeeding during infants' hospital stay and after discharge are critical to effectively manage preterm infants.

In addition to mothers' effort, the overall health status and feeding tolerance of preterm infants also influence breastfeeding ${ }^{[34]}$. In this study, feeding was performed by healthcare professionals during infants' hospital stay. With the involvement of parents in feeding post-discharge, the concerns and anxiety about feeding complications (abdominal distention, vomiting, choking, constipation, diarrhea, and hematochezia) increased significantly $\left(28.6 \%\right.$ vs. $\left.7.1 \% \triangle Z^{2}=10.957, p=0.001\right)$ and maintained, which is consistent with the findings of Callen $\mathrm{J}$ et al ${ }^{[34]}$.

Our study found that at post-discharge two weeks, the top challenge in breastfeeding associated with mothers was poor breastfeeding techniques. To improve breastfeeding skills, we recommend providing training on how to use human milk fortifier, adjust milk volume, clean breastfeeding devices, and practice direct breastfeeding. It is also important to train mothers on how to coordinate feeding rhythm with infants' needs as described by Ikonen and Palmer ${ }^{[15,35]}$. In addition, mothers should be instructed to recognize signs of hunger and to develop flexible feeding plans ${ }^{[36]}$.

\section{Limitations}

This was a single-center study. Although the number of participants was relatively small, the management and education of the mothers were highly consistent in this study. The GA range of the participating preterm infants was wide. Twins were also included in the analysis. Longer follow-up than CA of 3 months might further elucidate the breastfeeding status of mothers of preterm infants. In addition, in this study, frozen expressed milk was used to feed the infants. We did not investigate how frozen breast expressed milk could influence the breastfeeding duration and human milk proportion. 


\section{Conclusions}

Cesarean section, older mother age, and primiparous status were independent predictors of delayed lactogenesis II. Milk output volume on day 7 postpartum $>190 \mathrm{~mL} / \mathrm{d}$ and $>245 \mathrm{~mL} / \mathrm{d}$ predicted $>300 \mathrm{~mL}$ milk output at discharge and exclusive breastfeeding post-discharge, respectively. Difficulties in breastfeeding infants, poor breastfeeding technique, and insufficient milk output were the predominant challenges faced by mothers. Thus, interventions targeting to improve early postpartum lactation and trainings targeting to improve breastfeeding technique and reduce the difficulties in breastfeeding may increase breastfeeding adoption in mothers giving birth to preterm infants.

\section{Abbreviations}

CA 3m: 3 months of corrected age; MOM: mother's own milk; NICU: neonatal intensive care unit; GA: gestational age; CA: corrected age; HMF: Human milk fortifier; ROC: receiver operating characteristic.

\section{Declarations}

\section{Ethical approval and consent to participate:}

The study protocol was approved by the Institutional Review Board of Peking University First Hospital (project identification code: 2018-88; date of approval: May $\left.23^{\text {rd }}, 2018\right)$. Written informed consent was obtained from all participating mothers.

\section{Consent for publication}

Not applicable.

\section{Availability of data and materials}

The datasets generated and/or analysed during the current study are not publicly available for ethical reasons but are available from the corresponding author upon reasonable request.

\section{Competing interests}

None.

\section{Funding}

None.

\section{Authors' Contributions}

QF and DD designed the study. DD collected and interpreted the data and drafted the manuscript. QF critically revised the manuscript. All authors critically reviewed the manuscript and approved the final version of the manuscript as submitted. 


\section{Acknowledgments}

We would like to thank all the participating mothers.

\section{References}

1. Trend, S, Strunk, T, Hibbert, J, Kok, CH, Zhang, G, Doherty, DA, et al: Antimicrobial protein and Peptide concentrations and activity in human breast milk consumed by preterm infants at risk of late-onset neonatal sepsis. PLoS One 2015. 10(2): p. e0117038.

2. Serrao, F, Papacci, P, Costa, S, Giannantonio, C, Cota, F, Vento, G, et al: Effect of Early Expressed Human Milk on Insulin-Like Growth Factor 1 and Short-Term Outcomes in Preterm Infants. PLoS One 2016. 11(12): p. e0168139.

3. Cortez, J, Makker, K, Kraemer, DF, Neu, J, Sharma, R, and Hudak, ML: Maternal milk feedings reduce sepsis, necrotizing enterocolitis and improve outcomes of premature infants. J Perinato/ 2017. 38: p. 71.

4. Hair, AB, Peluso, AM, Hawthorne, KM, Perez, J, Smith, DP, Khan, JY, et al: Beyond Necrotizing Enterocolitis Prevention: Improving Outcomes with an Exclusive Human Milk-Based Diet. Breastfeed Med 2016. 11(2): p. 70-74.

5. Assad, M, Elliott, MJ, and Abraham, JH: Decreased cost and improved feeding tolerance in VLBW infants fed an exclusive human milk diet. J Perinatol 2016. 36(3): p. 216-220.

6. Johnson, TJ, Patra, K, Greene, MM, Hamilton, M, Dabrowski, E, Meier, PP, et al: NICU human milk dose and health care use after NICU discharge in very low birth weight infants. J Perinato/ 2019. 39(1): p. 120-128.

7. Boundy, EO, Perrine, CG, Nelson, JM, and Hamner, HC: Disparities in Hospital-Reported Breast Milk Use in Neonatal Intensive Care Units - United States, 2015. MMWR Morb Mortal Wkly Rep 2017. 66(48): p. 1313-1317.

8. Wilson, E, Edstedt Bonamy, AK, Bonet, M, Toome, L, Rodrigues, C, Howell, EA, et al: Room for improvement in breast milk feeding after very preterm birth in Europe: Results from the EPICE cohort. Matern Child Nutr 2018. 14(1).

9. Jiangsu Multicenter Study Collaborative Group for Breastmilk Feeding in Neonatal Intensive Care Units:

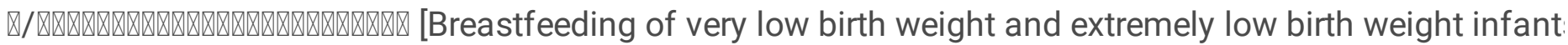

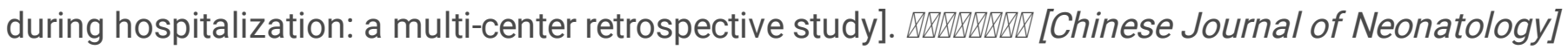
2020. 35(03): p. 164-168.

10. Hei, M, Gao, X, Li, Z, Zhang, Q, Li, J, Xia, S, et al: family integrated care model in neonatal intensive care unit]. 2018. 33(1).

11. Li, Z, Dong, M, Wang, D, and Valentine, CJ: infants between two hospitals in China and the United States]. Contemporary Pediatrics] 2014. 16(7): p. 691-695. 
12. Liu, F, Han, S, Yu, Z, Zhang, J, Chen, X, Wu, W, et al: quality improvement on breastfeeding rate in very low birth weight and extremely low birth weight

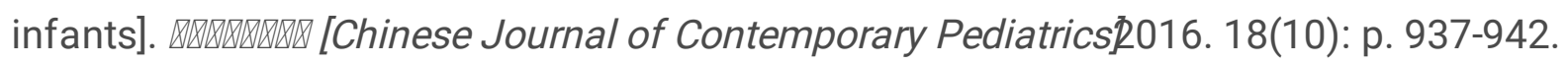

13. Cun, D, Zhang, K, and Yu, G: Cost Analysis of Operating a Human Milk Bank in China. J Hum Lact 2020: p. 890334419894551.

14. Parker, LA, Sullivan, S, Krueger, C, and Mueller, M: Association of timing of initiation of breastmilk expression on milk volume and timing of lactogenesis stage II among mothers of very low-birth-weight infants. Breastfeed Med 2015. 10(2): p. 84-91.

15. Ikonen, R, Paavilainen, E, and Kaunonen, M: Preterm Infants' Mothers' Experiences With Milk Expression and Breastfeeding: An Integrative Review. Adv Neonatal Care 2015. 15(6): p. 394-406.

16. Pinchevski-Kadir, S, Shust-Barequet, S, Zajicek, M, Leibovich, M, Strauss, T, Leibovitch, L, et al: Direct Feeding at the Breast Is Associated with Breast Milk Feeding Duration among Preterm Infants. Nutrients 2017. 9(11).

17. Chapman, DJ, Perez-Escamilla, R: Maternal perception of the onset of lactation is a valid, public health indicator of lactogenesis stage II. J Nutr 2000. 130(12): p. 2972-2980.

18. Meier, PP, Engstrom, JL, Janes, JE, Jegier, BJ, and Loera, F: Breast pump suction patterns that mimic the human infant during breastfeeding: greater milk output in less time spent pumping for breast pumpdependent mothers with premature infants. J Perinato/ 2012. 32(2): p. 103-110.

19. Dewey, KG, Nommsen-Rivers, LA, Heinig, MJ, and Cohen, RJ: Risk factors for suboptimal infant breastfeeding behavior, delayed onset of lactation, and excess neonatal weight loss. Pediatrics 2003. 112(3 Pt 1): p. 607-619.

20. Parker, LA, Sullivan, S, Krueger, C, Kelechi, T, and Mueller, M: Effect of early breast milk expression on milk volume and timing of lactogenesis stage II among mothers of very low birth weight infants: a pilot study. J Perinatol 2012. 32(3): p. 205-209.

21. Yu, X, Li, J, Lin, X, and Luan, D: Association between Delayed Lactogenesis and Early Milk Volume among Mothers of Preterm Infants. Asian Nurs Res (Korean Soc Nurs Sci) 2019. 13(2): p. 93-98.

22. Brownell, E, Howard, CR, Lawrence, RA, and Dozier, AM: Delayed onset lactogenesis II predicts the cessation of any or exclusive breastfeeding. J Pediatr 2012. 161(4): p. 608-614.

23. Liu, P, Qiao, L, Xu, F, Zhang, M, Wang, Y, and Binns, CW: Factors associated with breastfeeding duration: a 30-month cohort study in northwest China. J Hum Lact 2013. 29(2): p. 253-259.

24. Chapman, DJ, Perez-Escamilla, R: Does delayed perception of the onset of lactation shorten breastfeeding duration? J Hum Lact 1999. 15(2): p. 107-111; quiz 137-109.

25. Zhu, P, Hao, J, Jiang, X, Huang, K, and Tao, F: New insight into onset of lactation: mediating the negative effect of multiple perinatal biopsychosocial stress on breastfeeding duration. Breastfeed Med 2013. 8: p. 151-158.

26. Murase, M, Nommsen-Rivers, L, Morrow, AL, Hatsuno, M, Mizuno, K, Taki, M, et al: Predictors of low milk volume among mothers who delivered preterm. J Hum Lact 2014. 30(4): p. 425-435. 
27. Nommsen-Rivers, LA, Chantry, CJ, Peerson, JM, Cohen, RJ, and Dewey, KG: Delayed onset of lactogenesis among first-time mothers is related to maternal obesity and factors associated with ineffective breastfeeding. Am J Clin Nutr 2010. 92(3): p. 574-584.

28. Chapman, DJ, Perez-Escamilla, R: Identification of risk factors for delayed onset of lactation. J Am Diet Assoc 1999. 99(4): p. 450-454; quiz 455-456.

29. Flacking, R, Nyqvist, KH, Ewald, U, and Wallin, L: Long-term duration of breastfeeding in Swedish low birth weight infants. J Hum Lact 2003. 19(2): p. 157-165.

30. Omarsdottir, S, Adling, A, Bonamy, AK, Legnevall, L, Tessma, MK, and Vanpee, M: Predictors of sustained maternal milk feeds in extremely preterm infants. $J$ Perinatol 2015. 35(5): p. 367-372.

31. Meio, M, Villela, LD, Gomes Junior, S, Tovar, CM, and Moreira, MEL: Breastfeeding of preterm newborn infants following hospital discharge: follow-up during the first year of life. Cien Saude Colet 2018. 23(7): p. 2403-2412.

32. Wang, Y, Briere, $\mathrm{CE}, \mathrm{Xu}, \mathrm{W}$, and Cong, X: Factors Affecting Breastfeeding Outcomes at Six Months in Preterm Infants. J Hum Lact 2018: p. 890334418771307.

33. Vohr, BR, Poindexter, BB, Dusick, AM, McKinley, LT, Higgins, RD, Langer, JC, et al: Persistent beneficial effects of breast milk ingested in the neonatal intensive care unit on outcomes of extremely low birth weight infants at 30 months of age. Pediatrics 2007. 120(4): p. e953-959.

34. Callen, J, Pinelli, J, Atkinson, S, and Saigal, S: Qualitative analysis of barriers to breastfeeding in verylow-birthweight infants in the hospital and postdischarge. Adv Neonatal Care 2005. 5(2): p. 93-103.

35. Palmer, L, Ericson, J: A qualitative study on the breastfeeding experience of mothers of preterm infants in the first 12 months after birth. Int Breastfeed J 2019. 14: p. 35.

36. Gianni, ML, Bezze, EN, Sannino, P, Baro, M, Roggero, P, Muscolo, S, et al: Maternal views on facilitators of and barriers to breastfeeding preterm infants. BMC Pediatr 2018. 18(1): p. 283.

\section{Figures}


Screened: 157 infants with a gestation age $<37$ weeks from 124 mothers were admitted to the NICU between May $26^{\mathrm{t}}$ and October $31^{\mathrm{s}}, 2018$.

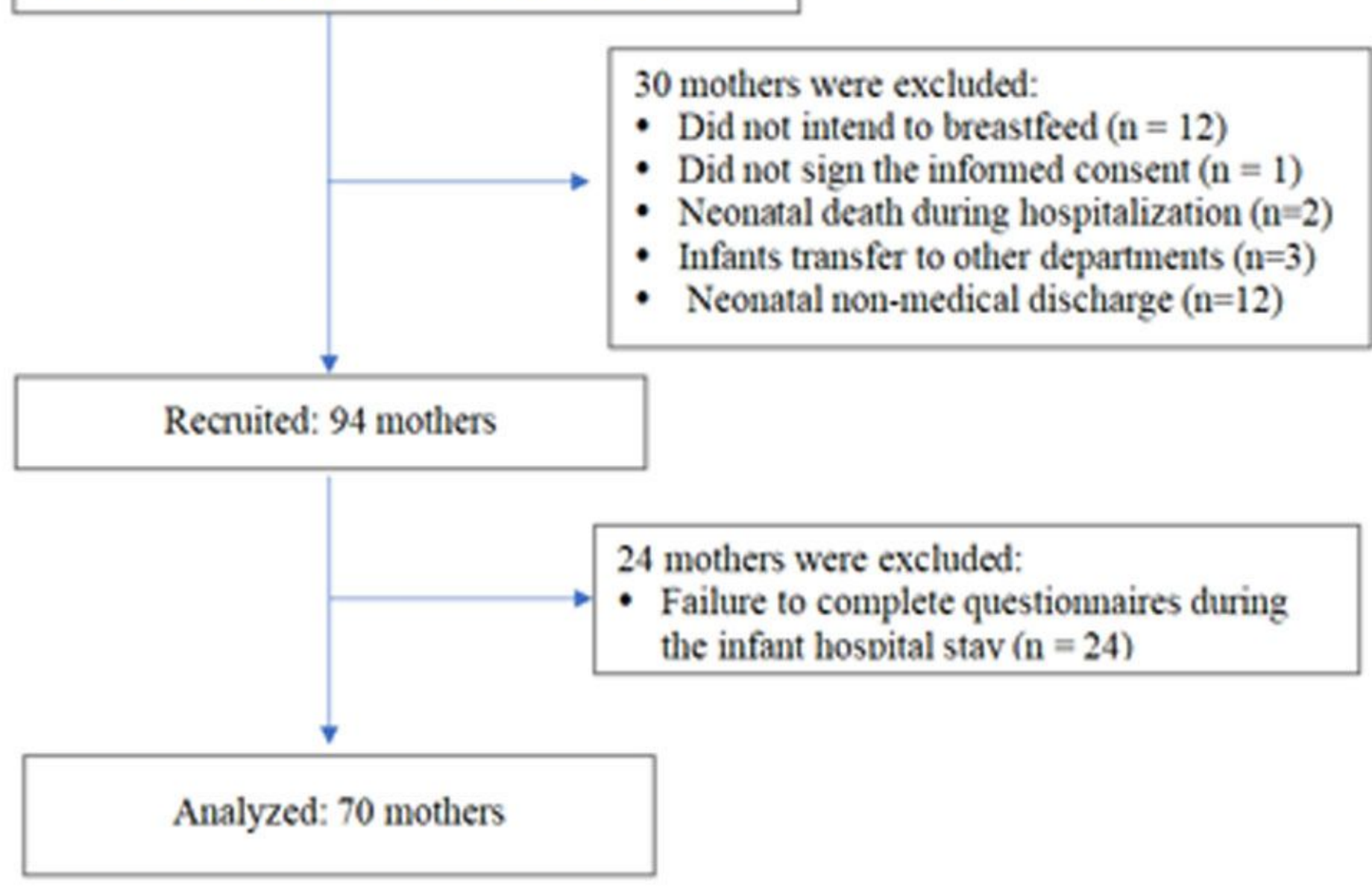

Figure 1

Flow Chart of Study Participants 
A

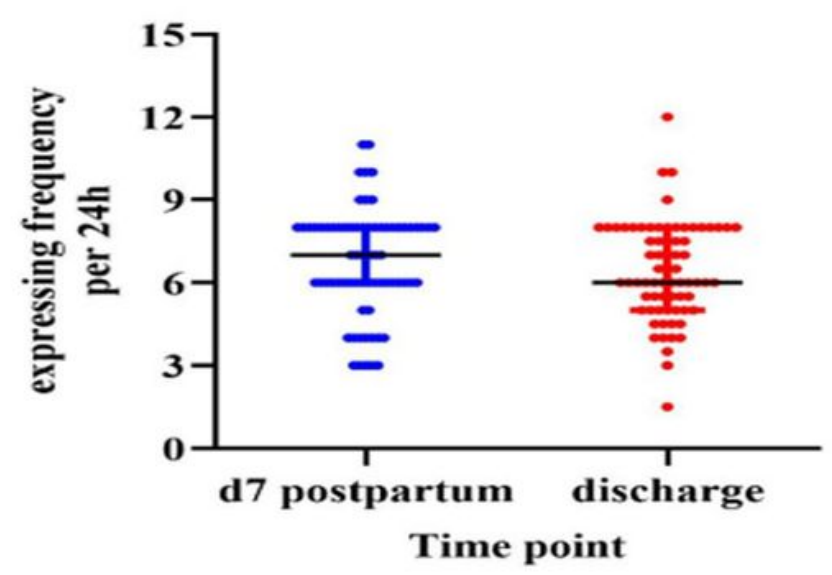

B
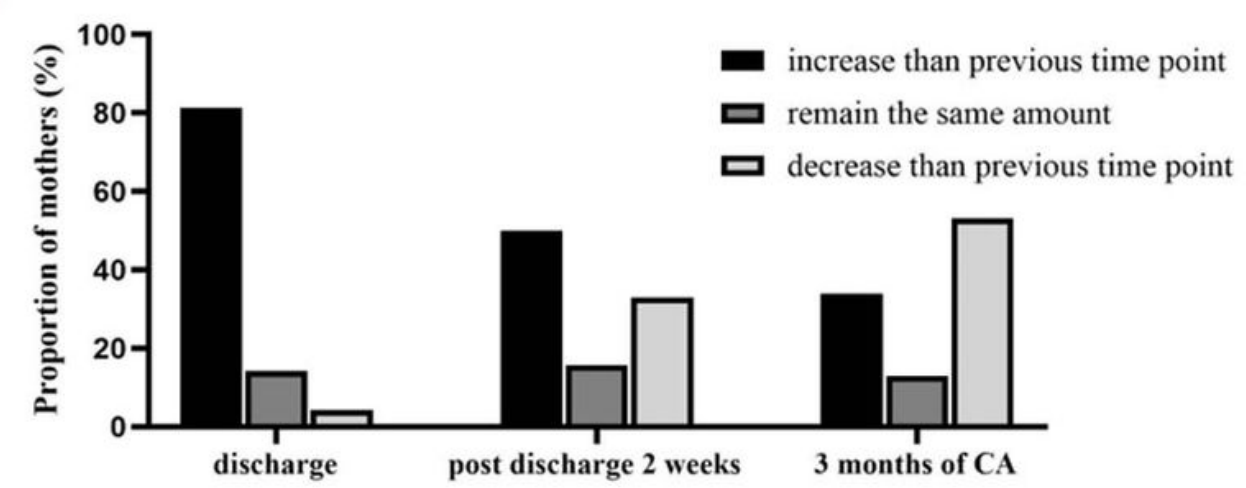

Time point

ROC for predicting exclusive breastfeeding at post-discharge two weeks

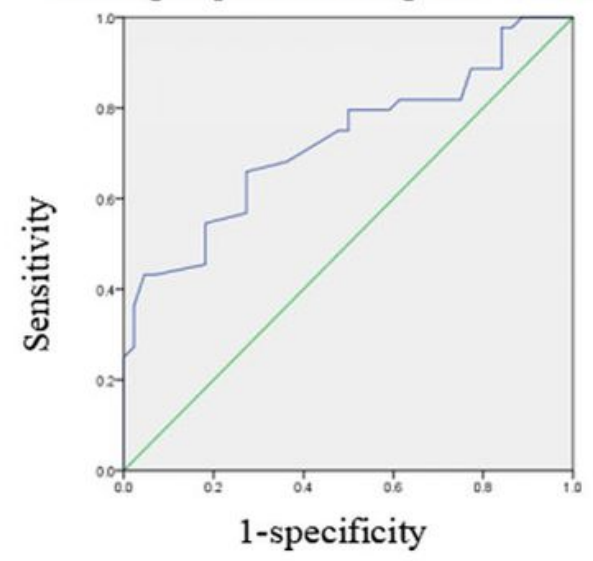

ROC for predicting milk output $>$ $300 \mathrm{~mL} / \mathrm{d}$ at discharge

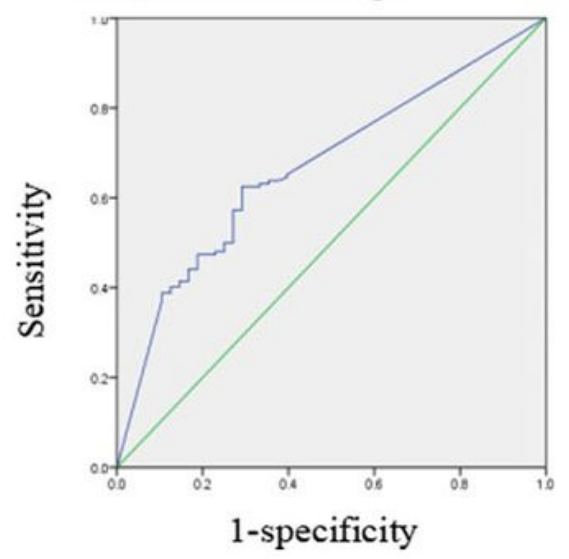

Figure 2

Expressing Frequency and Changes and Prediction Value of Milk Output A. Expressing frequency on day 7 postpartum and discharge of infants. B. The proportions of mothers with increased, same, or decreased milk output compared with the previous time point. The time points of milk output measurement were day 7 postpartum, the time of discharge of infants, post-discharge 2 weeks, and CA of 3 months. C. The predictive values of expressed milk output volume at day 7 postpartum. Output volume at day 7 postpartum reaching $190 \mathrm{~mL} / \mathrm{d}$ was an optimal threshold for predicting (ROC) expressed milk volume more than 300ml/d at 
discharge (right). Output volume at day 7 postpartum reaching $245 \mathrm{~mL} / \mathrm{d}$ predicted the possibility of exclusive breastfeeding at post-discharge two weeks (left).

\section{Supplementary Files}

This is a list of supplementary files associated with this preprint. Click to download.

- SupplementaryTable.docx 